\title{
Ultrafast Coherent Terahertz Spectroscopy in High Magnetic Fields and Directed Energy Flows in Quantum Dot Assemblies
}

\author{
S.A. Crooker, T. Barrick \\ National High Magnetic Field Laboratory, Los Alamos, NM 87545, USA \\ J.A. Hollingsworth AND V.I. Klimov \\ Chemistry Division, Los Alamos, NM 87545, USA
}

\begin{abstract}
We describe the design, construction, and use of fiber-coupled terahertz antennas for performing ultrafast coherent $\mathrm{THz}$ spectroscopy directly in the cryogenic bore of high-field magnets. With an aim towards measuring the high-frequency (100 $\mathrm{GHz}$ to $2000 \mathrm{GHz}$ ) complex conductivity of correlated electron materials in the regime of low temperatures and high magnetic fields, these miniature $\mathrm{THz}$ emitters and receivers are demonstrated to work down to $1.5 \mathrm{~K}$ and up to $18 \mathrm{~T}$, for eventual use in higher-field magnets. Results from a variety of semiconducting and superconducting samples are presented. This paper also describes a separate effort towards achieving coupling between colloidal semiconductor nanocrystal quantum dots, wherein we realize and study inter-dot communication via resonant (Förster) energy transfer. We present studies of the dynamics of resonant energy transfer in monodisperse and energy gradient (layered) assemblies of CdSe nanocrystal quantum dots. Time- and spectrally-resolved photoluminescence data directly reveal the energy-dependent transfer rate of excitons from smaller to larger dots. Results from layered nanocrystal quantum dot assemblies demonstrate unidirectional energy flows, a first step towards artificial light-harvesting structures. Lastly, time-resolved studies at millikelvin temperatures elucidate the nature of ground-state "dark" excitons in these quantum dots.
\end{abstract}

PACS numbers:

\section{THz spectroscopy in high magnetic fields}

Time-domain terahertz spectroscopy is an established technique for the measurement of high-frequency conductivity, typically in the range between $100 \mathrm{GHz}$ 
and $\approx 3000 \mathrm{GHz}$. Pioneered in the late 1980s [1], "table-top" terahertz spectrometers employing photoconductive antennas gated by ultrafast optical pulses have been used to study a wide range of material systems, including semiconductors and dielectrics, normal and high- $T_{\mathrm{C}}$ superconductors, liquids, flames, and gases [2]. This $\mathrm{THz}$ frequency range lies between that which is readily accessible by microwave cavity techniques (on the low frequency side), and Fourier-transform infrared spectroscopies (on the high frequency side). Further, these frequencies correspond to energies between $0.4 \mathrm{meV}$ and $\approx 12 \mathrm{meV}$, or alternatively, temperatures between $4 \mathrm{~K}$ and $140 \mathrm{~K}$ and magnetic fields between $4 \mathrm{~T}$ and $100 \mathrm{~T}$.

This is precisely the energy, temperature, and in particular the magnetic field scale relevant to many novel correlated-electron systems of interest today, including high- $T_{\mathrm{c}}$ superconductors (where the upper critical field $H_{\mathrm{c} 2}$ may be tens or even hundreds of $\mathrm{T}$ ), heavy-fermion and Kondo-insulating materials (where, e.g., the Kondo spin/charge gap in $\mathrm{Ce}_{3} \mathrm{Bi}_{4} \mathrm{Pt}_{3}$ may be closed above $30 \mathrm{~T}$ ), colossal magnetoresistive manganites (melting of charge/orbital order at high fields), 2D electron gases (composite fermion dynamics in the high-field fractional quantum Hall regime), and organic metals (novel field-induced superconductivity above $17 \mathrm{~T}$ ). Thus it is of keen interest to perform measurements of the complex $\mathrm{THz}$ conductivity not only in the regime of low temperatures, but also at high magnetic fields. However, the conventional "table-top" transmission terahertz spectrometer is a rather involved and physically large setup, typically utilizing several micropositioning stages to align the $\mathrm{THz}$ antennas with respect to the free-space laser beams, and off-axis parabolic optics to collimate and focus the terahertz pulses over short distances. These traditional methods work extremely well, but are not compatible with high-field magnets $(10-60 \mathrm{~T})$, which are generally solenoids with narrow, cryogenic bores accessible primarily via meters-long experimental probes.

To this end we have developed extremely sensitive, miniaturized, optical fiber-coupled $\mathrm{THz}$ emitters and receivers for remote use directly in the low-temperature bore of a high-field (dc or pulsed) magnet [3]. These devices permit ultrafast, coherent, time-domain $\mathrm{THz}$ transmission spectroscopy of samples in the frequency range between $100 \mathrm{GHz}$ and $\approx 2000 \mathrm{GHz}$. Due to the coherent nature of the detection (both amplitude and phase of the THz electric field are measured), the complex conductivity may be directly evaluated without the need for Kramers-Kronig analyses. The gated nature of the detection permits high signal-to-noise data with minimal $(\approx 2 \mathrm{~mW})$ optical power input and (where necessary), the ability to acquire complete spectra in tens of milliseconds. The primary challenges of this project include maintaining sub-micron alignment between fiber and antenna upon repeated thermal cycling, achieving ultrafast $(<200 \mathrm{fs})$ optical pulses at the end of tens of meters of single-mode optical fiber, and obtaining complete time-domain scans with high signal:noise using only milliwatts of optical power, no lock-in detection, and (for pulsed magnets) only $\approx 100 \mathrm{~ms}$ of integration time. 
Using photoconductive antennas, wide-band width $\mathrm{THz}$ pulses may only be generated (and detected) by gating the antennas with ultrafast optical pulses, and for this reason it is necessary to compensate for the positive group-velocity-dispersion (GVD) of optical fibers so that fast optical pulses may be obtained at the ends of long fibers. Normal silica optical fiber exhibits a GVD of roughly $120 \mathrm{fs} / \mathrm{m}-\mathrm{nm}$ at $800 \mathrm{~nm}$, so that without compensation, a $100 \mathrm{fs}$ input optical pulse with a band width of $10 \mathrm{~nm}$ broadens, in the best case, to $>20 \mathrm{ps}$ after a typical $20 \mathrm{~m}$ length of fiber. Such a lengthy optical pulse is useless for generating or detecting $\mathrm{THz}$ radiation. Thus it is necessary to precompensate and impose a negative chirp on the optical pulses before launching into the optical fibers, so that the optical pulses shorten in time as they travel through the fiber and achieve a minimum value at the $\mathrm{THz}$ devices.

The experimental schematic for $\mathrm{THz}$ transmission spectroscopy in high-field magnets is shown in Fig. 1, along with a photo of the actual THz probe. Ultrafast optical pulses (100 fs, centered at $800 \mathrm{~nm}$ ) from a Ti:sapphire laser are directed to a 2-grating pulse stretcher, which imparts a negative chirp (blue wavelengths

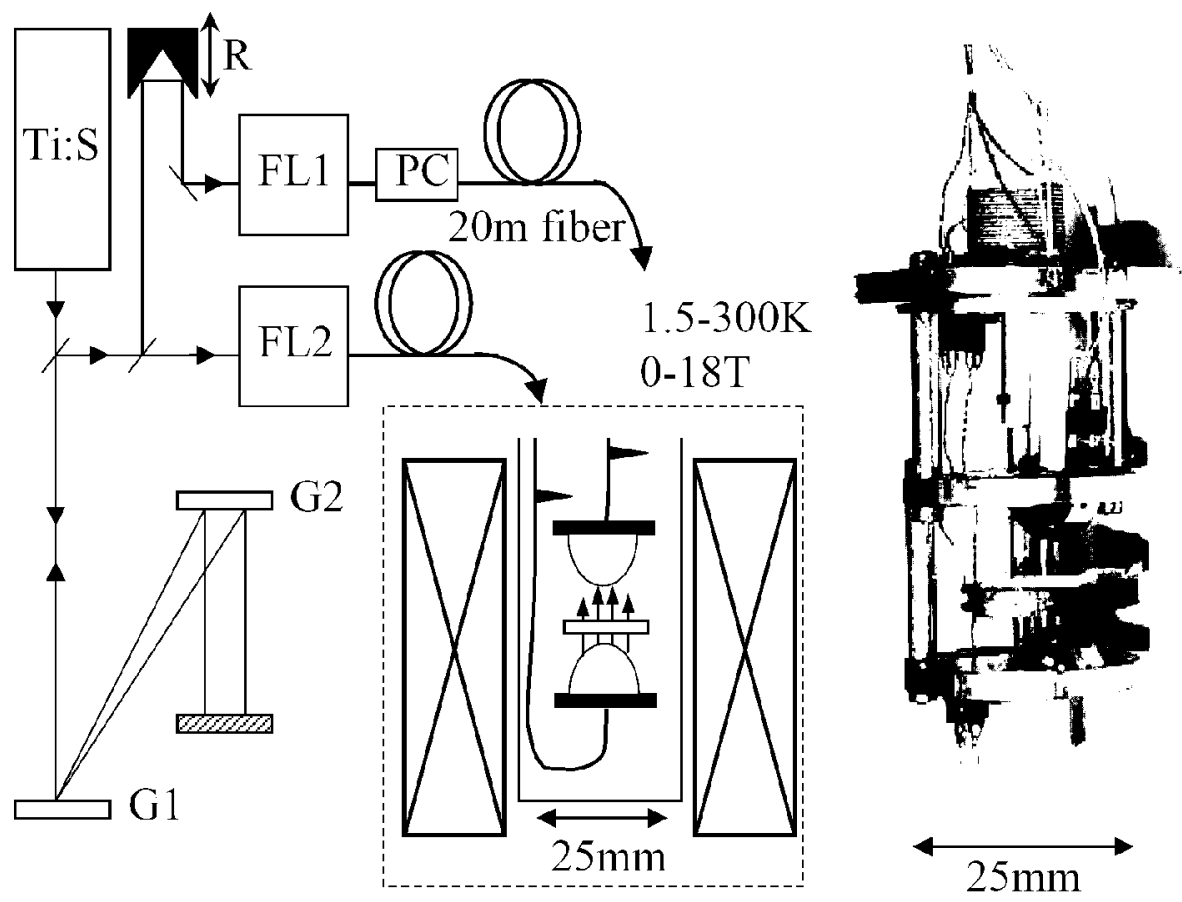

Fig. 1. Experimental $\mathrm{THz}$ schematic. Ultrafast optical pulses are pre-chirped (stretched) by gratings $G 1$ and $G 2$, and coupled into fibers by fiber launchers $F L 1$ and FL2. Pulses achieve minimum temporal width (and highest peak intensity) at the photoconductive $\mathrm{THz}$ emitter and receiver, which are located in the cryogenic bore of a high-field magnet. On right, a photo of the THz probe. Adapted from Ref. [3]. 
leading red wavelengths) onto the pulses. The magnitude of the negative chirp, tuned via the separation between the two gratings $G 1$ and $G 2$, is chosen to optimally compensate for the intrinsic positive GVD of optical fiber, and thus depends on the laser wavelength and the length of fiber used. For typical fiber lengths of $20 \mathrm{~m}$, the $100 \mathrm{fs}$, transform-limited pulses are stretched to approximately $24 \mathrm{ps}$. After leaving the pulse stretcher, the negatively-chirped pulse train is equally split into an "emitter" and a "receiver" beam. The former is launched directly into a single-mode optical fiber, while the latter is delayed by a scanning retroreflector before being launched into another single-mode fiber of equal length. As the optical pulses travel from the laboratory to the low-temperature probe housing the $\mathrm{THz}$ antennas, their temporal width decreases due to the positive GVD of the fiber, achieving a minimum pulse width directly at the lithographically-defined photoconductive antennas.

In an experiment, the sample is positioned between the $\mathrm{THz}$ emitter and receiver in the cryogenic bore of the magnet. An ultrafast optical pulse promotes mobile carriers in the biased (20-30 V) strip-line emitter, and the subsequent current surge generates a burst of broad-band $\mathrm{THz}$ radiation which is coupled into free space through a collimating silicon hyperhemispherical substrate lens. After passing through the sample, the $\mathrm{THz}$ pulse is focused onto the strip-line receiver by another substrate lens, where the "instantaneous" $\mathrm{THz}$ electric field is gated by a second ultrafast optical pulse, generating a measurable current. The complete time-dependent $\mathrm{THz}$ electric field is mapped by rapidly scanning the time delay between the excitation and gating optical pulses, and the externally-amplified current signal is sent directly to digitizers. The $\mathrm{THz}$ emitter and receiver are mounted facing one another with a gap of roughly $1 \mathrm{~cm}$. A rotating copper sample stage enables the sample to be moved out of the $\mathrm{THz}$ beam path, so that a reference scan may be taken at each new temperature or field (crucial for quantitative interpretation of data, since the exact $\mathrm{THz}$ wave forms are field- and temperature-dependent). The entire probe is $25 \mathrm{~mm}$ in diameter. The $\mathrm{THz}$ strip-line antennas themselves are based on $20 \mu \mathrm{m}$ wide, $1 \mathrm{~cm}$ long, titanium-gold lines separated by $50 \mu \mathrm{m}$. The antennas also incorporate micron-scale features, matched to the mode-field diameter of the optical fiber, to maximize their efficiency and sensitivity.

Figure 2 shows characteristic data taken on a very high mobility 2-dimensional electron gas (2DEG) (mobility $10^{7} \mathrm{~cm}^{2} /(\mathrm{V} \mathrm{s})$ ) formed at a GaAs/AlGaAs heterojunction, where the sample and antennas are immersed in superfluid helium at $1.6 \mathrm{~K}$. Here, the raw power spectrum of the transmitted THz pulse is shown in the low-field regime where the electron cyclotron energy falls within the $\mathrm{THz}$ detection band width. Clear oscillations in the time-domain data (not shown) correspond to the observed cyclotron absorption resonance, which evolves with the expected behavior: $\omega_{\mathrm{c}}=e B / \hbar m^{*} c=1.73 \mathrm{meV} / \mathrm{T}=420 \mathrm{GHz} / \mathrm{T}$ (the additional ripples in the power spectrum are an artifact arising from a multiple reflection of the $\mathrm{THz}$ pulse which appears $\approx 12 \mathrm{ps}$ later in the time domain, and which may be avoided 


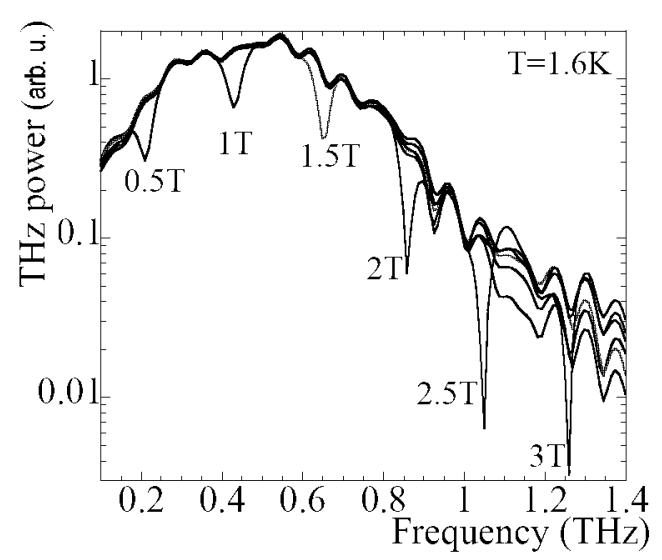

Fig. 2. Low-field dependence of the power spectrum of the raw time-domain THz data upon passage through a high mobility GaAs/AlGaAs 2D electron gas at $1.6 \mathrm{~K}$. Absorption resonances correspond to cyclotron motion. Adapted from Ref. [3].

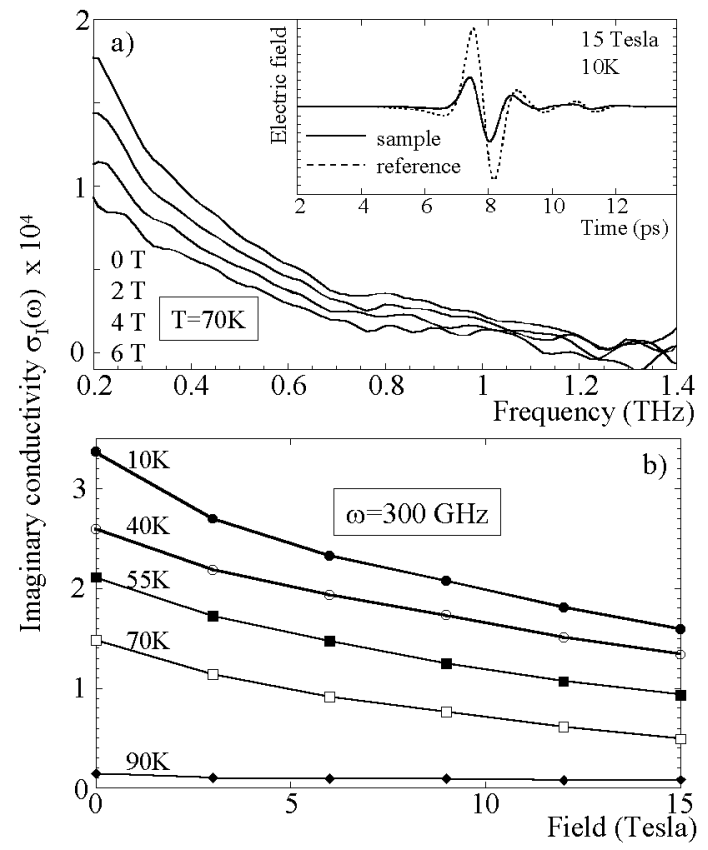

Fig. 3. (a) THz imaginary conductivity of a $\mathrm{YBCO}$ film $\left(T_{\mathrm{c}} \approx 85 \mathrm{~K}\right)$, taken in the cryogenic bore of a $17 \mathrm{~T}$ superconducting solenoid. The $1 / \omega$ conductivity decreases with increasing magnetic field, indicating the suppression of superconductivity. Inset: raw data traces of the measured electric field of the THz pulse. (b) A $300 \mathrm{GHz}$ cross-section of the field- and temperature-dependent imaginary conductivity of YBCO. 
by stacking additional "dummy" wafers of GaAs onto the back of the 2DEG sample). Using $\mathrm{THz}$ spectroscopy, very sensitive density- and field-dependent studies of the $\mathrm{THz}$ conductivity of ultraclean $2 \mathrm{D}$ electron systems in the fractional quantum Hall regime may be performed, providing deeper insight into the dynamics and interactions of composite fermions.

Figure 3 shows the complex conductivity on a very different system; namely a thin film of superconducting YBCO (the $50 \mathrm{~nm}$ thick film is grown on a $\mathrm{MgO}$ substrate, and has $T_{\mathrm{c}} \approx 85 \mathrm{~K}$ ). The data show the imaginary part of the measured $\mathrm{THz}$ conductivity. The inset shows the raw data (the electric field of the $\mathrm{THz}$ pulse) acquired in the experiment, when the $\mathrm{THz}$ pulse passes through the YBCO film, and also through a reference MgO substrate. From these data (taken at $15 \mathrm{~T}$ and $10 \mathrm{~K}$ ) the complex transmission coefficient, $t(\omega)$, may be directly measured for all frequencies between $\approx 200-1400 \mathrm{GHz}$ (see Fig. 3a). Below $T_{\mathrm{C}}$, the $1 / \omega$ conductivity from the Drude-like response of superconducting particles with infinite scattering time is observed. With increasing magnetic field, the conductivity is observed to decrease, indicating the field-induced suppression of phase coherent superconductivity. Figure $3 \mathrm{~b}$ shows the measured imaginary conductivity of $\mathrm{YBCO}$ at $300 \mathrm{GHz}$, as a function of magnetic field and at the temperatures indicated. Here, the expected suppression of superconductivity with increasing field and temperature is clearly seen.

\section{Time-resolved spectroscopy of nanocrystal quantum dots}

Communication, coupling, and coherence between quantum dots are central themes in numerous scientific efforts of present physical and technological interest. In the limit of large numbers of coupled dots, colloidal semiconductor nanocrystal quantum dots (NQDs) are promising building blocks for the bottom-up assembly of macroscopic "artificial materials" having engineered functionality. Recently, we investigated the coupling between quantum dots via long-range dipolar interactions, which allow inter-dot communication via resonant (Förster) energy transfer. Unidirectional transfer of excitons between quantum dots, a first step towards artificial light-harvesting structures, was directly observed in a series of time-resolved PL experiments on dense films and engineered bilayers of CdSe dots [4].

Figure 4 shows time-resolved PL decays from a sample of nominally monodisperse $1.24 \mathrm{~nm}$ CdSe dots, measured via time-correlated single photon counting using a frequency-doubled, pulse-picked ultrafast Ti:sapphire laser. The film's $200 \mathrm{meV}$ wide photoluminescence (PL) emission spectrum (see inset) results from the $\approx 8 \%$ dot size dispersion within the sample - smaller and larger quantum dots radiate towards higher and lower energies, respectively. Measuring the PL decay at particular wavelengths therefore allows investigation of exciton dynamics from dots of specific size. Isolated dots in solution exhibit the same $24 \mathrm{~ns}$ decay, regardless of detection wavelength. However, when the dots are packed together 


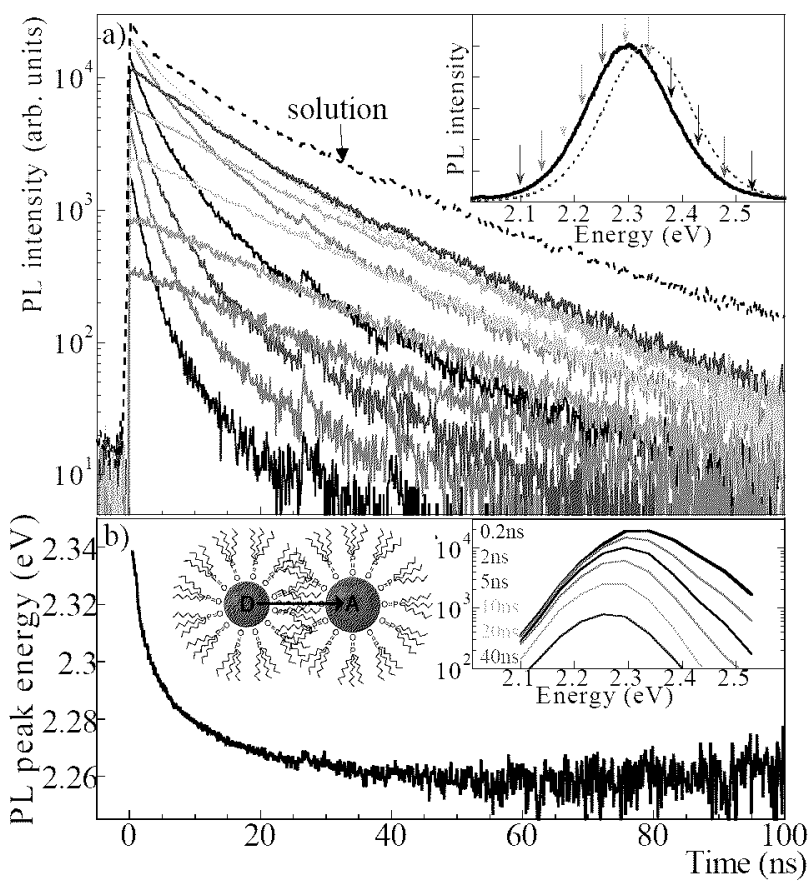

Fig. 4. (a) Time-resolved photoluminescence decays from a dense film of nominally 12.4 $\AA$ CdSe quantum dots at the energies specified in the inset. Inset: cw PL spectra from dense film (solid) and original solution (dashed), showing redshift. (b) Dynamic redshift of the PL peak, as excitons transfer from donor to acceptor dots. Inset: PL spectra at the specified times. Adapted from Ref. [4].

in a dense film, the PL dynamics measured at shorter wavelengths (smaller dots) becomes very rapid, of order $\approx 1$ ns. Conversely, at longer wavelengths (larger dots), the PL decay becomes quite slow, even exhibiting a slight upturn at short times. This behavior indicates that excitons are flowing from smaller (donor) dots to larger (acceptor) dots well after the initial photoexcitation. From these data one may derive "instantaneous" spectra to elucidate the dynamic PL redshift, shown in Fig. 4b. The emission peak redshifts rapidly during the first $10 \mathrm{~ns}$, and beyond $30 \mathrm{~ns}$ is roughly constant, indicating that energy transfer has effectively ceased all excitons have migrated to larger dots from which further transfer is suppressed by the vanishing probability of even-larger neighboring dots.

In comparison with some of Nature's most efficient energy transfer complexes - chlorophylls - the data suggest that inter-dot energy transfer can approach picosecond time scales in structurally optimized systems where the emission energy of donor dots overlaps optimally with the absorption energy of nearby acceptor dots. To this end, monolayer-by-monolayer assembly of quantum dots was performed to create an energy- (size-) gradient structure, shown schematically in Fig. 5. "Instan- 


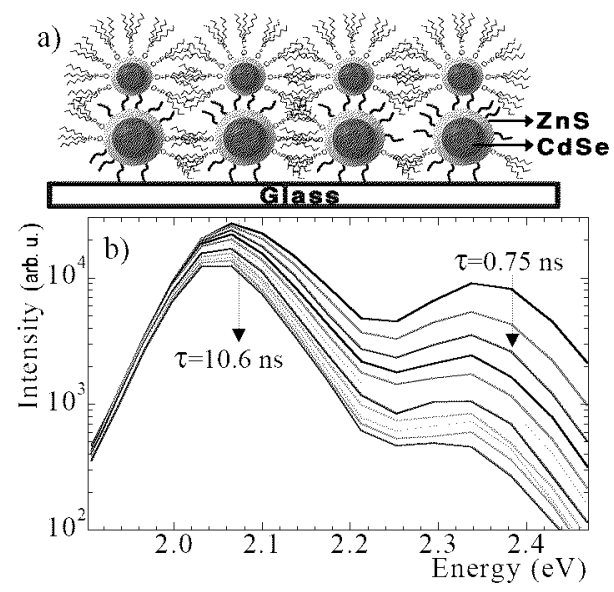

Fig. 5. (a) Schematic of NQD energy-gradient bilayer for light harvesting - $13 \AA$ dots on $20.5 \AA$ dots. (b) "Instantaneous" PL spectra at 500 ps intervals (from $0-5 \mathrm{~ns}$ ) showing rapid collapse of emission from $13 \AA$ dots. Adapted from Ref. [4].

taneous" spectra taken at 500 ps intervals reveal the nearly complete depletion of excitons from the monolayer of small quantum dots within 5 ns, but only a small decrease in the monolayer of large dots, indicating efficient energy transfer from small to large dots in the vertical direction (in solution, both large and small dots exhibit long $\approx 20$ ns decays). Photoluminescence decays from the small dots are very rapid $(750 \mathrm{ps})$, which is over twice as fast as compared with the monodisperse dots of Fig. 4, despite a larger interdot separation, and a much smaller number of possible neighbors due to the $2 \mathrm{D}$ bilayer nature of the structure. Thus by ensuring donor/acceptor spectral overlap, and intentionally placing acceptors near donor dots, the rate of energy transfer is enhanced, and the direction of energy flow can be influenced.

In a related experiment [5], time-resolved PL methods at low temperatures allow study of the intrinsic radiative lifetime $\left(\tau_{\mathrm{R}}\right)$ in CdSe quantum dots, wherein it is known that $\tau_{\mathrm{R}}$ far exceeds that of their bulk counterpart $\left(\tau_{\mathrm{R}} \approx 1 \mu \mathrm{s}, \mathrm{vs} . \approx 1 \mathrm{~ns}\right.$ in bulk CdSe at $4 \mathrm{~K}$ ). These long lifetimes result from the dot's intrinsic crystal/shape anisotropy, and confinement-enhanced electron-hole exchange energy. These effects lift the spin degeneracy of the band-edge exciton such that the ground state is, in fact, the exciton with net spin projection $J=2$, which is formally forbidden from direct radiative recombination (photons having spin 1), and is therefore optically inactive. This "dark" ground state lies well below the nearest optically active "bright" ( $J=1)$ exciton. The bright-dark energy gap, $\Delta=1-15 \mathrm{meV}$, scales inversely with dot size [6]. Thus, when the thermal energy $k_{\mathrm{B}} T<\Delta$, excitons are largely frozen in the dark state and no remaining decay channels exist. Within this framework, $\tau_{\mathbf{R}}$ should increase exponentially with decreasing temperature, raising the intriguing possibility of long-term "storage" of excitons in cold dots. 


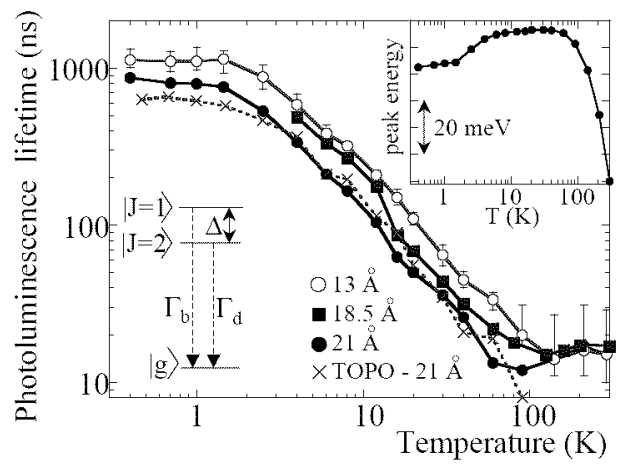

Fig. 6. Measured radiative lifetime ( $\log -\log$ scale) of CdSe quantum dots with radius $13,18.5$, and $21 \AA$, showing saturation $<2 \mathrm{~K}$ at $\approx 1 \mu \mathrm{s}$. Inset: peak emission energy vs. $T$ from $13 \AA$ dots, showing redshift $<4 \mathrm{~K}$ due to LO phonon coupling. Adapted from Ref. [5].

In this work we investigated $\tau_{\mathrm{R}}(T)$ over a very broad temperature range $(380 \mathrm{mK}$ to $300 \mathrm{~K})$ in a series of colloidal CdSe quantum dots. Our goal was to determine the dependence of exciton lifetime on temperature, dot size, and surface passivation. Figure 6 shows $\tau_{\mathbf{R}}$ in all the NQD samples, on a log-log scale where the rapid drop at intermediate temperatures is clear. For temperatures above $20 \mathrm{~K}$, overall trends may be modelled by thermal activation between "dark" and higher-lying "bright" exciton states. Most striking, though, is the low-temperature saturation of $\tau_{\mathrm{R}}$ below $2 \mathrm{~K}$, observed in all samples regardless of size or surface passivation. The observed lifetime saturation is surprising within the context of a dark-exciton ground state where, as discussed above, $\tau_{\mathrm{R}}$ should continue to increase with lower temperatures. Because the overall PL quantum yield does not change appreciably below $20 \mathrm{~K}$ (not shown), these data point towards the existence of an intrinsic radiative decay channel which, below $2 \mathrm{~K}$, evidently imposes a fundamental limit of roughly $1 \mu$ s on the storage time of excitons in these CdSe dots. This low-temperature decay channel likely involves recombination of dark excitons assisted by an angular momentum conserving LO phonon, where direct evidence for phonon assisted recombination at low temperatures is seen (inset, Fig. 6) in the sizable redshift of the PL peak energy below $4 \mathrm{~K}$.

\section{Acknowledgments}

This work was supported by the NHMFL In-House Research Program, and the Los Alamos LDRD programs. 


\section{References}

[1] P.R. Smith, D.H. Auston, M.C. Nuss, IEEE J. Quantum Electron. QE-24, 255 (1988); C. Fattinger, D. Grischkowsky, Appl. Phys. Lett. 54, 490 (1989).

[2] See, e.g., papers by M.C. Nuss, and by D. Grischkowsky.

[3] S.A. Crooker, Rev. Sci. Instrum. 73, 3258 (2002).

[4] S.A. Crooker, J.A. Hollingsworth, S. Tretiak, V.I. Klimov, Phys. Rev. Lett. 89, $186802(2002)$

[5] S.A. Crooker, T. Barrick, J.A. Hollingsworth, V.I. Klimov, Appl. Phys, Lett. 82, 2793 (2003).

[6] Al.L. Efros, M. Rosen, M. Kuno, M. Nirmal, D.J. Norris, M. Bawendi, Phys. Rev. $B$ 54, 4843 (1996). 